\title{
Non-alcoholic fatty liver disease in patients with psoriasis: therapeutic implications
}

\author{
Elzbieta H. Klujszoํㅜㄹ Piotr Parcheta' ${ }^{1}$ Anna B. Witkowska², Beata Krecisz ${ }^{3}$ \\ ${ }^{1}$ Department of Dermatology, Regional Hospital, Kielce, Poland \\ ${ }^{2}$ Graduate of Medical University, Szeged, Hungary \\ ${ }^{3}$ Faculty of Medicine and Health Science, Jan Kochanowski University, Kielce, Poland
}

Adv Dermatol Allergol 2020; XXXVII (4): 468-474

DOI: https://doi.org/10.5114/ada.2019.83983

\begin{abstract}
Non-alcoholic fatty liver disease (NAFLD) is the most common liver pathology in the western countries. Psoriatic patients are at higher risk of having NAFLD, and at higher risk of experiencing a more severe form of the disease with poorer outcomes. The components of the metabolic syndrome - obesity, lipid abnormalities, hypertension, and type 2 diabetes - significantly correlate with NAFLD progression. The inflammatory state present in psoriasis plays a significant role in development of NAFLD and the metabolic syndrome. All patients with psoriasis and insulin resistance and risk factors for metabolic syndrome should also been screened for NAFLD, and planning of the treatment options should always take into consideration the possible risks related to the liver, especially in patients with NAFLD.
\end{abstract}

Key words: psoriasis, liver disease, methotrexate, biologic agents.

\section{Introduction}

Psoriasis is a chronic, inflammatory disease caused by genetic, environmental and immunologic factors. Skin and joints are the most affected sites; however, there is a growing number of comorbidities leading to serious health and psychological implications [1, 2]. During the last decade, there has been increased scientific research about the association between psoriasis and other diseases including cardio-vascular, metabolic, gastrointestinal, liver, kidney diseases, depression, tumours, infections, and uveitis [3-7].

Recently, there has been growing interest in non-alcoholic fatty liver disease (NAFLD) and its higher prevalence among patients with psoriasis. The aim of this review is to show the link between NAFLD and psoriasis and the implications in applying safe therapy.

\section{Non-alcoholic fatty liver disease}

NAFLD is defined as excessive liver fat accumulation in absence of other reasons for these accumulations, such as excessive alcohol consumption, hereditary or acquired diseases such as Wilson's disease, lipodystrophy, abetalipoproteinemia, starvation, as well as exogenous factors such as medications. Excessive alcohol intake, for clinical purposes, is defined as current or recent alcohol consumption of more than 21 portions per week
( 1 portion $=10 \mathrm{~g}$ of ethanol) for men, and more than 14 portions for women [8]. In the case of NAFDL, its overall pathology is related to metabolic derangements, particularly insulin resistance (IR). Primary NAFLD can only be diagnosed when other causes of liver disease and excessive ethanol consumption have been excluded.

Currently, NAFLD is thought to be the liver manifestation of the metabolic syndrome [9]. There are well-known conditions that are associated with NAFLD in particular obesity, type 2 diabetes (T2DM), hypertension, and dyslipidaemia. New emerging conditions are sleep apnoea, colorectal cancer, osteoporosis, psoriasis, endocrinopathies, and polycystic ovary syndrome independent of obesity [10]. Studies conducted in Eastern Asia suggest that the serum uric acid (SUA) level is highly related to NAFLD. Data from a study on the US population also confirmed the increased risk of NAFLD associated with a high serum level of SUA. This increased risk is probably independent of conventional NAFLD risk factors [11]. The prevalence of NAFLD may vary according to age, sex, and ethnicity [12]. In fact, both the prevalence of NAFLD and stage of liver disease appear to increase with age. Although controversial, male sex has been considered a risk factor for NAFLD. Furthermore, the prevalence of NAFLD in men is 2 times higher than in women [13].

The authors from Shanghai, China (study on 439 patients) have found that patients with psoriasis onset

Address for correspondence: Elzbieta H. Klujszo PhD, MD, Department of Dermatology, Regional Hospital, 7 Radiowa St, 25-375 Kielce, Poland, e-mail: klujszoe@gmail.com Received: 5.12.2017, accepted: 25.10.2018. 
before the age of 40 have a significantly higher prevalence of NAFLD than patients with late onset of psoriasis (74.2\% and $40.4 \%$, respectively) [14].

Diet may affect modulation of liver fat accumulation, regulation of antioxidant activity, insulin sensitivity and postprandial triglyceride metabolism. Physical activity may modulate liver fat content through weight loss, but may also directly reduce oxidative stress [15-18].

The management of NAFLD and non-alcoholic steatohepatitis (NASH) prevention should consist of treating liver disease as well as the associated metabolic comorbidities such as obesity, hyperlipidaemia, IR, and T2DM. To date, lifestyle modification, through exercise and weight loss efforts, has been the cornerstone of management of NAFLD. There is no standard pharmacological therapy for NAFLD or NASH [10].

NAFLD covers a wide spectrum of pathophysiological features - from a simple hepatosteatosis called nonalcoholic fatty liver (NAFL), through steatohepatitis NASH, liver fibrosis, and liver cirrhosis with its potential complications, including hepatocellular carcinoma [19].

The underlying mechanism for the development and progression of NAFLD is complex and multifactorial. One of the first hypotheses regarding progression of simple fatty liver to steatohepatitis was the "two hit" model, proposed by Day and James in 1998 [20]. This model suggests that the first "hit", the development of steatosis [21], sensitizes the liver to the second "hit" - oxidative stress and subsequent lipid peroxidation, proinflammatory cytokines, adipokine release and mitochondrial dysfunction promote hepatic injury, inflammation and fibrosis, leading to apoptosis, necroinflammation and ultimately fibrosis and cirrhosis, through activation of hepatic stellate cells [22]. The pathogenesis of NAFLD and its progression is a complex process, which cannot be completely explained by the "two hit" hypothesis. A number of diverse parallel processes contribute to the development of steatosis and liver inflammation. The "multiple hit" hypothesis considers multiple insults acting together on genetically predisposed subjects to induce NAFLD and provides a more accurate explanation of NAFLD pathogenesis. Such hits include insulin resistance, hormones secreted from the adipose tissue, nutritional factors, gut microbiota and genetic and epigenetic factors [23].

The world epidemiological studies concerning NAFLD, NAFL and NASH prevalence are not conclusive. It is estimated that NAFLD is the most frequent liver pathology in Western countries and affects $17-46 \%$ of the population [11], and NASH 3-5\% [8]. These data depend on the region, the examined population and methodology. The risk factors for NAFLD are obesity and type 2 diabetes [8]. Even though most of the NAFLD cases are not reported because of its symptomless nature [24], we can currently observe an alarming rise of NAFLD frequency in the global population.

The NAFLD diagnosis has to be confirmed by imaging tests or liver biopsy. The diagnostic value of imaging tests such as ultrasonography (USG), computed tomography $(\mathrm{CT})$, magnetic resonance $(\mathrm{MRI})$ is very high for NAFLD (sensitivity and specificity > 90\%) [25].

USG confirms most of the cases of average and severe steatosis if it affects more than $30 \%$ of hepatocytes [26]. Unfortunately, imaging techniques are not able to differentiate between NAFL and NASH. The gold standard of NASH diagnosis is liver punch biopsy [8]. Because of the risk of progression of the relatively benign NAFL into NASH with liver fibrosis and cirrhosis, there is a need for a non-invasive monitoring method for these patients.

A routine biochemistry panel is recommended: albumin, bilirubin, alanine aminotransferase (ALT), aspartate aminotransferase (AST), $\gamma$-glutamyltransferase (GGT) as well as blood morphology. Most commonly one can observe an increase in the AST and ALT levels, usually not exceeding 5 times the upper limits [27]. Nevertheless, sometimes the activity of liver enzymes does not correlate with NAFLD severity [28]. Identifying the presence and severity of the liver fibrosis in patients with NAFLD is of major importance. Non-invasive assessment of the stage of fibrosis is done by evaluating the rigidity of the liver by means of transient elastography. This method is used for evaluating the liver stiffness. The fibrosis correlates well with liver stiffness [29]. Because the elastography results have a higher rate of false-positive than false-negative results, they cannot be used for making clinically significant decisions [30]. The European societies for liver disease, diabetes and obesity (European Association for the Study of the Liver (EASL), European Association for the Study of Diabetes (EASD) and European Association for the Study of Obesity (EASO)) have published new guidelines for NAFLD management [30].

According to these regulations, all patients with insulin resistance and risk factors for metabolic syndrome should be screened for NAFLD, by the use of abdominal USG and liver biochemistry panels. If NAFLD is confirmed by the USG, any increase in ALT, AST or GGT requires further diagnostics. If no liver enzyme abnormalities are present, it is recommended to evaluate the risk of fibrosis using serum fibrosis markers such an "NAFLD fibrosis score" test (NFS). The test takes into account 6 parameters (age, body mass index (BMI), glucose level, platelet number, albumin concentration, AST/ALT ratio) and it is calculated with the use of a formula available online (http://nafldscore.com/) [31]. Results suggesting a low risk require follow-up every 2 years while any medium to high risk require further specialist referral [24]. Psoriasis patients with risk factors for metabolic syndrome treated by dermatologists should be evaluated based on these regulations.

\section{Non-alcoholic fatty liver disease and psoriasis}

NAFLD is considered to be a liver manifestation of the metabolic syndrome [9], and psoriasis has a strong connection with this syndrome [32], thus the frequent 
co-presence of the 2 diseases is unsurprising. Mantovani et al. [33] have suggested a new definition of a hepatodermal axis, highlighting the importance of the mutual pathophysiological mechanisms of the two diseases. Ganzetti et al. have extended this definition by cardiovascular diseases (CVD), finding mutual inflammatory biomarkers for psoriasis, NAFLD and CVD [34].

Recent studies have shown a correlation between psoriasis, particularly its severe form and psoriatic arthritis with NAFLD [35-38]. Gisondi et al. [35] have shown that patients with psoriasis have twice as high a risk of NAFLD (confirmed by an ultrasound). In addition, NAFLD was more frequent in patients with severe psoriasis (when estimated by PASI score). In another retrospective study Miele et al. [36] have found NAFLD in 59.2\% of psoriatic patients.

In a study done by Roberts et al. that included 103 patients with psoriasis and psoriatic arthritis, the prevalence of NAFLD was estimated to be $47 \%$ (confirmed by USG), and prevalence of NASH to be $22 \%$ (confirmed by punch biopsy) [39].

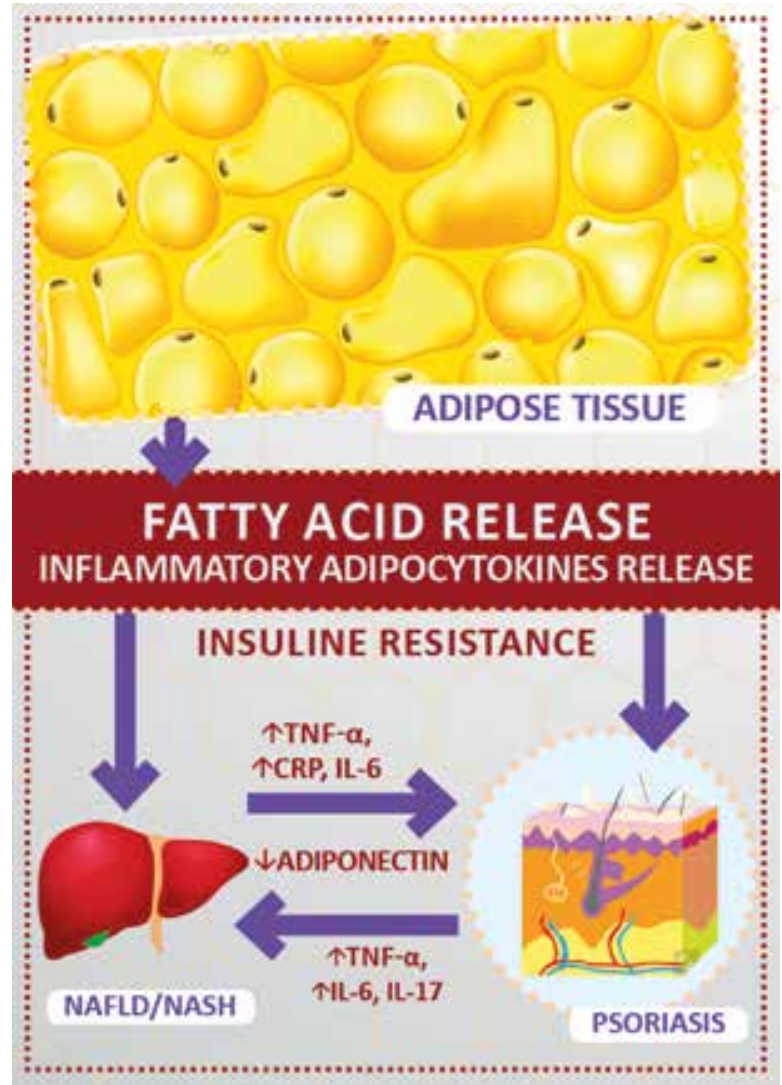

Figure 1. Hypothesized connections between the skin, adipose tissue (WAT) and liver damage - a "vicious circle". Modified from Mantovani et al. [33]

TNF - tumour necrosis factor, CRP - C-reactive protein, IL-6 interleukin 6, IL-17 - interleukin 17, NAFLD - non-alcoholic fatty liver disease, NASH - non-alcoholic steatohepatitis.
In a meta-analysis of 7 different studies done in 2015: patients with psoriasis ( $n=267761$ ) had a higher risk of NAFLD when compared to a control group with no psoriasis - odds ratio $(O R)=2.15$. The risk of NAFLD was significantly higher for patients with psoriatic arthritis $(n=$ $505, \mathrm{OR}=2.25$ ) and for patients with moderate to severe psoriasis when compared to patients with mild psoriasis $(\mathrm{OR}=2.07)$ [40].

The above data show that in psoriatic patients there is an increased risk of severe hepatic complications. Thus, there is a need for meticulous liver monitoring, especially for patients subjected to chronic therapies [33].

\section{Mutual pathophysiological mechanisms for psoriasis and non-alcoholic fatty liver disease}

Psoriasis, as well as NAFLD, is a multifactorial disease. Common mechanisms responsible for psoriasis and NAFLD are mediated by pro-inflammatory cytokines.

The same mediators responsible for psoriasis and atherosclerosis may promote the liver steatosis and worsen both the NAFLD and psoriasis [33] (Figure 1).

The NAFLD pathogenesis has not been yet fully elicited. It is thought that insulin resistance plays a major role in initiating the disease process and, recently, obesity and adipokines (bioactive molecules released by the adipose tissue) have been suggested as well. A possible interaction between visceral fat, fatty liver and psoriatic skin has also been investigated [33].

White adipose tissue (WAT) is the source of proinflammatory cytokines such as TNF- $\alpha$, interleukin-1, interleukin-6, leptin, visfatin, chemerin, and resistin. At the same time there is a decreased production of adiponectin (an anti-inflammatory cytokine), by the adipose tissue. Obesity, type 2 diabetes, hypertension, NAFLD correlate with decreased levels of adiponectin. Psoriatic patients also have a reduced level of adiponectin. Even lower levels of adiponectin were observed in patients with both psoriasis and NAFLD compared with patients affected with psoriasis without hepatic involvement [41]. This aspect could be linked to the adiponectin-mediated suppression of T-helper(Th)1 type cytokines which prevent psoriasis-prone individuals from developing the disease until the imbalance of pro- and anti-inflammatory cytokines observed in patients with NAFLD antagonize these effects [41].

Pro-inflammatory adipokines such as interleukin-6 (IL-6), leptin, and resistin seem to play a role in the pathogenesis of both NAFLD and psoriasis. Tumor necrosis factor $\alpha$ TNF- $\alpha$ plays a role in NAFLD pathogenesis via promoting insulin resistance (stopping the insulin signalling at the receptor level) and lipolysis stimulation with free fatty acids release [42]. Induction of liver fibrosis by TNF- $\alpha$ by Kupffer cell activation has also been described [43]. TNF- $\alpha$ is a key cytokine in the pathogenesis of psoriasis. Imbalances between the pro- and anti-inflammatory 
cytokines promote the progression of liver disease. WATproduced adipokine excess may influence the severity of psoriasis by increased keratinocyte proliferation, Th1 activation, angiogenesis and adhesion molecules activation in the skin [24].

It is highly probable that the mediators released by the fatty, inflamed liver have a negative effect on the severity of psoriasis by promoting keratinocyte proliferation [41].

Moreover, the multiple immunological and metabolic derangements mutually enhanced both diseases [33].

\section{The influence of psoriasis therapy on NAFLD}

There are many options available for the treatment of psoriasis. In general, they are classified as topical, systemic and phototherapy.

The data previously mentioned in this article highlight connections between psoriasis and metabolic derangements and NAFLD. Thus, the safety of patients with psoriasis and NAFLD depends on the right choice of systemic therapy.

\section{Methotrexate}

For more than 40 years, methotrexate (MTX) has been an effective and widely used medication in the therapy of moderate to severe psoriasis. It has antiproliferative effects, but it also works by suppressing the inflammation $[44,45]$ as well as inhibition of the Th1/ Th17 pathways [46].

MTX induces the synthesis of pro-fibrotic factors in hepatocytes and astrocytes by depleting the cells' folate storages [47, 48]. MTX-induced liver damage is histologically similar to changes that occur in NAFLD [49]. The first description of the MTX-induced liver fibrosis case in a patient treated for psoriasis was done in 1964 [50]. Together with rising frequency of MTX usage, there is a higher level of liver toxicity. The first American Association of Dermatology (AAD) guidelines for monitoring MTX-treated psoriasis patients recommended pre-treatment liver biopsy followed by series of biopsies during the treatment [51].

Currently, routine liver biopsies during MTX-treatment are not recommended. The last 2009 AAD recommendations recognize an increased liver fibrosis risk in patients with psoriasis and co-existing obesity, type 2 diabetes, excessive alcohol intake or a pre-existing liver pathology [52]. The newest EADV recommendations from 2015 assess the risk of liver fibrosis/cirrhosis after MTX use as moderately small, as long as appropriate screening and monitoring procedures are being used [53].

What is interesting to note is that there are more patients with MTX-induced liver injury with psoriasis than with rheumatoid arthritis, according to the literature [54]. Thus, there is a different perception of the risk of hepatotoxicity and its monitoring between rheumatologists and dermatologists [55]. The authors of the paper discussing the relation between the MTX-hepatotoxicity and NAFLD hypothesize that the higher frequency of hepatic fibrosis in patients with psoriasis than in patients with rheumatoid arthritis is due to the fact that the metabolic syndrome and NAFLD is overall less frequent in patients with rheumatoid arthritis [51].

The presence of NAFLD in patients with psoriasis increases the risk of hepatotoxicity of MTX, and therefore these patients should be evaluated more thoroughly before treatment induction. It is recommended that the risk-benefit ratio of MTX therapy in such patients should be calculated on an individual basis. It is important to recognize $\mathrm{NASH}$, especially $\mathrm{NASH}$ with the presence of fibrosis [51].

\section{Cyclosporine A}

Cyclosporine A, used for the treatment of moderate to severe psoriasis may induce hepatotoxicity. This may be a direct or an indirect process, by means of provoking an atherogenic dyslipidaemia, which promotes NAFLD. However, this is not common and usually affects liver transplant recipients [33].

\section{Acitretin}

Hepatic adverse reactions have been described during the treatment with acitretin, which is one of the gold standards of the conventional systemic treatment of psoriasis. Approximately $25 \%$ of patients can develop elevation of liver enzymes, what is usually transient, and severe liver toxicity is rare [56]. The role of acitretin in the process of liver fibrosis is unequivocal. The retinoic acid may induce the fibrosis process as well as accelerate its development. However, most sources identify acitretin as a liver protective agent. An et al. have shown that the combination therapy with MTX and acitretin results in less MTX-induced liver fibrosis without an increased risk of hepatotoxicity [57].

\section{Biological treatment}

The biological treatment options seem to be safer for the liver. Although there are several biological agents, three classes of biological treatments for psoriasis are available in practice. The first group includes tumour necrosis factor $\alpha$ inhibitors (TNFi): etanercept, infliximab and adalimumab. The second group includes ustekinum$\mathrm{ab}$, antibodies directed against interleukin IL-12 and IL-23 (IL-12/IL-23). The third group includes interleukin-17 antagonists: secukinumab and ixekizumab [58].

In general, the biological treatments seem not to have a negative effect on the liver enzymes and other biochemical parameters when compared to conventional therapies. In the long term, the incidence of withdrawal from the treatment caused by side effects was higher in the group of patients treated with the conventional drugs than in the patients with biological treatments $[59,60]$.

TNF- $\alpha$ plays a major role in the induction of the inflammatory process in NAFLD. The role of TNFi in limit- 
ing the inflammatory process in animal models makes it plausible to introduce a TNF- $\alpha$ inhibitors therapy for liver diseases such as NASH in people. Unfortunately, the results of such trials are not conclusive - etanercepttreated patients with liver alcohol damage were shown to have a higher risk of death when compared to a placebo group (related to a higher risk of infection) [61]. The improvement in the liver enzymes profile is, however, observed after treatment with infliximab and adalimumab in patients with NAFLD and psoriasis [24]. The influence of TNFi on insulin resistance, which is one of the major factors responsible for fatty liver, is not yet determined. Treatment with etanercept was found to be beneficial on glucose homeostasis in obese patients [62]. In a metaanalysis of patients with rheumatoid arthritis, a beneficial effect of TNFi on insulin resistance was observed [63]. This beneficial effect was not, however, shown in patients with psoriasis [64]. It is considered that, in general, treatment with TNFi does not have a negative effect on the lipid profile, and clinically important dyslipidaemia is rare. On the other hand, a significant increase in body mass can be observed mainly in patients with psoriasis undergoing treatment with TNFi [53]. That was not observed in patients who were treated with ustekinumab.

The recently published literature recognizes the possibility of NAFLD as a negative side effect of TNFi therapy. NAFLD developing and progression was found in 8 patients during TNFi therapy, with no increase of body weight in most patients and normalization of ALT after therapy cessation [65]. In another study of patients with NAFLD and psoriasis treated with TNFi, a worsening of the NAFLD was noted in patients in whom there was no resolution of joint symptoms after therapy cessation [66]. The earlier studies describing liver damage related to the TNFi therapy usually describe drug-induced liver injury (DILI) or an autoimmune hepatitis. The risk of developing such conditions is very small. DILI was described after treatment with all clinically available TNFis, especially after infliximab. The spectrum of the DILI syndrome consisted of conditions such as elevation of liver enzymes (usually resolving on its own) and cholestatic hepatitis [61]. There is little information on the effects of IL-12/23 and IL-17 on the liver. In the studies of ustekinumab no elevation of liver enzymes was seen. An increase in IL-12 activity in the liver of patients with NAFLD may signify a shift towards NASH. It is thus reasonable to hypothesize that therapy that hinders cytokine-related pathways may have a beneficial effect in such patients. Thus, therapy for psoriasis with the use of anti-IL-12/IL-23 for patients who were previously treated with hepatotoxic drugs or who have liver pathologies, such as NAFLD seems to be safer than other options [67].

\section{Conclusions}

Psoriatic patients, particularly with its severe form and coexisting metabolic syndrome are at higher risk of developing NASH with all its consequences, what has to be taken into consideration while planning long-term treatment therapies.

\section{Conflict of interest}

The authors declare no conflict of interest.

\section{References}

1. Di Meglio P, Nestle FO. Immunopathogenesis of Psoriasis. In: Clinical and Basic Immunodermatology. Gaspari A, Tyring S, Kaplan D (eds). Springer, Cham 2017.

2. Davidovici BB, Sattar N, Prinz JC, et al. Psoriasis and systemic inflammatory diseases: potential mechanistic links between skin disease and co-morbid conditions. J Invest Dermatol 2010; 130: 1785-96.

3. Takeshita J, Grewal S, Langan SM, et al. Psoriasis and comorbid diseases: implications for management. J Am Acad Dermatol 2017; 76: 393-403.

4. Yeung H, Takeshita J, Mehta NN, et al. Psoriasis severity and the prevalence of major medical comorbidity: a populationbased study. JAMA Dermatol 2013; 149: 1173-9.

5. Armstrong AW, Harskamp CT, Armstrong EJ. Psoriasis and metabolic syndrome: a systematic review and meta-analysis of observational studies. J Am Acad Dermatol 2013; 68: 654-62.

6. Ma C, Harskamp C, Armstrong E, Armstrong A. The association between psoriasis and dyslipidaemia: a systematic review. Br J Dermatol 2013; 168: 486-95.

7. Miller IM, Ellervik S, Yazdanyar S, Jemec GBE. Meta-analysis of psoriasis, cardiovascular disease, and associated risk factors. J Am Acad Dermatol 2013; 69: 1014-24.

8. Chalasani N, Younossi Z, Lavine JE, et al. The diagnosis and management of non-alcoholic fatty liver disease: practice Guideline by the American Association for the Study of Liver Diseases, American College of Gastroenterology, and the American Gastroenterological Association. Hepatology 2012; 55: 2005-23.

9. Kotronen A, Yki-Järvinen $\mathrm{H}$. Fatty liver: a novel component of the metabolic syndrome. Arterioscler Thromb Vasc Biol 2008; 28: 27-38.

10. Chalasani N, Younossi Z, Lavine JE, et al. The diagnosis and management of nonalcoholic fatty liver disease: practice guidance from the American Association for the Study of Liver Diseases. Hepatology 2018; 67: 328-57.

11. Le MH, Devaki P, Ha NB, et al. Prevalence of non-alcoholic fatty liver disease and risk factors for advanced fibrosis and mortality in the United States. PLoS One 2017; 12: e0173499.

12. Fattahi MR, Niknam R, Safarpour A, Sepehrimanesh M, Lotfi $M$. The prevalence of metabolic syndrome in non-alcoholic fatty liver disease: a population-based study. Middle East J Dig Dis 2016; 8: 131-7.

13. Zelber-Sagi S, Nitzan-Kaluski D, Halpern Z, Oren R. Prevalence of primary non-alcoholic fatty liver disease in a population-based study and its association with biochemical and anthropometric measures. Liver Int 2006; 26: 856-63.

14. Xin X, Su L, Gao Y, Ding Y. The Prevalence of nonalcoholic fatty liver disease and related metabolic comorbidities was associated with age at onset of moderate to severe plaque psoriasis: a cross-sectional study. PLoS One 2017; 12: e0169952.

15. de Wit NJW, Afman LA, Mensink M, Müller M. Phenotyping the effect of diet on non-alcoholic fatty liver disease. J Hepatol 2012; 57: 1370-3. 
16. Perseghin G, Lattuada G, De Cobelli F, et al. Habitual physical activity is associated with intrahepatic fat content in humans. Diabetes Care 2007; 30: 683-8.

17. Zelber-Sagi S, Nitzan-Kaluski D, Goldsmith R, et al. Role of leisure-time physical activity in nonalcoholic fatty liver disease: a population-based study. Hepatology 2008; 48: 1791-8.

18. Keating SE, Hackett DA, George J, Johnson NA. Exercise and non-alcoholic fatty liver disease: a systematic review and meta-analysis. J Hepatol 2012; 57: 157-66.

19. Mehta R, Younossi Z. Natural history of nonalcoholic fatty liver disease. Clin Liv Dis 2012; 22: 11-21.

20. Day CP, James OFW. Steatohepatitis: a tale of two "hits"? Gastroenterology 1998; 114: 842-5.

21. Schreuder TCMA, Verwer BJ, van Nieuwkerk CMJ, Mulder CJJ. Nonalcoholic fatty liver disease: an overview of current insights in pathogenesis, diagnosis and treatment. World J Gastroenterol 2008; 14: 2474-86.

22. Albano E, Mottaran E, Occhino G, et al. Role of oxidative stress in the progression of non-alcoholic steatosis. Aliment Pharmacol Ther 2005; 22 Suppl 2: 71-3.

23. Buzzetti E, Pinzani M, Tsochatzis EA. The multiple-hit pathogenesis of non-alcoholic fatty liver disease (NAFLD). Metabolism 2016; 65: 1038-48.

24. Carrascosa JM, Bonanad C, Dauden E, et al. Psoriasis and nonalcoholic fatty liver disease. Actas Dermo-Sifiliográficas 2017; 108: 506-14.

25. Lee SS, Park SH. Radiologic evaluation of nonalcoholic fatty liver disease. World J Gastroenterol 2014; 20: 7392-402.

26. Hernaez R, Lazo M, Bonekamp S, et al. Diagnostic accuracy and reliability of ultrasonography for the detection of fatty liver: a meta-analysis. Hepatology 2011; 54: 1082-90.

27. Cheah MC, McCullough AJ, Goh GB. Current modalities of fibrosis assessment in non-alcoholic fatty liver disease. J Clin Transl Hepatol 2017; 5: 261-71.

28. Mofrad P, Contos MJ, Haque M, et al. Clinical and histologic spectrum of nonalcoholic fatty liver disease associated with normal ALT values. Hepatology 2003; 37: 1286-92.

29. Zaleska-Dorobisz U, Pawlus A, Kucharska M, Inglot M. Elastografia SWE w ocenie włóknienia wątroby. Postepy Hig Med Dosw 2015; 69: 221-6.

30. EASL-EASD-EASO Clinical Practice Guidelines for the management of non-alcoholic fatty liver disease. J Hepatol 2016; 64: 1388-402

31. EASL-ALEH Clinical Practice Guidelines: non-invasive tests for evaluation of liver disease severity and prognosis. J Hepatol 2015; 63: 237-64.

32. Gottlieb AB, Chao C, Dann F. Psoriasis comorbidities. J Dermatol Treat 2008; 19: 5-21.

33. Mantovani A, Gisondi P, Lonardo A, Targher G. Relationship between non-alcoholic fatty liver disease and psoriasis: a novel hepato-dermal axis? Int J Mol Sci 2016; 17: 217.

34. Ganzetti G, Campanati A, Molinelli E, Offidani A. Psoriasis, non-alcoholic fatty liver disease, and cardiovascular disease: three different diseases on a unique background. World J Cardiol 2016; 8: 120-31.

35. Gisondi P, Targher G, Zoppini G, Girolomoni G. Non-alcoholic fatty liver disease in patients with chronic plaque psoriasis. Jepatol 2009; 51: 758-64.

36. Miele L, Vallone S, Cefalo C, et al. Prevalence, characteristics and severity of non-alcoholic fatty liver disease in patients with chronic plaque psoriasis. J Hepatol 2009; 51: 778-86.

37. van der Voort EAM, Koehler EM, Dowlatshahi EA, et al. Psoriasis is independently associated with nonalcoholic fatty liver disease in patients 55 years old or older: results from a population-based study. J Am Acad Dermatol 2014; 70: 517-24.

38. Gisondi P, Barba E, Girolomoni G. Non-alcoholic fatty liver disease fibrosis score in patients with psoriasis. J Eur Acad Dermatol Venereol 2016; 30: 282-7.

39. Roberts KK, Cochet AE, Lamb PB, et al. The prevalence of NAFLD and NASH among patients with psoriasis in a tertiary care dermatology and rheumatology clinic. Aliment Pharmacol Ther 2015; 41: 293-300.

40. Candia R, Ruiz A, Torres-Robles R, et al. Risk of non-alcoholic fatty liver disease in patients with psoriasis: a systematic review and meta-analysis. J Eur Acad Dermatol Venereol 2015; 29: 656-62

41. Ganzetti G, Campanati A, Offidani A. Non-alcoholic fatty liver disease and psoriasis: so far, so near. World J Hepatol 2015; 7: 315-26.

42. Carter-Kent C, Zein NN, Feldstein AE. Cytokines in the pathogenesis of fatty liver and disease progression to steatohepatitis: implications for treatment. Am J Gastroenterol 2008; 103: $1036-42$.

43. Tsochatzis EA, Papatheodoridis GV, Archimandritis AJ. Adipokines in nonalcoholic steatohepatitis: from pathogenesis to implications in diagnosis and therapy. Mediators Inflamm 2009; 2009: 831670.

44. Chan ES, Cronstein BN. Methotrexate - how does it really work? Nature Rev Rheumatol 2010; 6: 175-8.

45. Cronstein B. How does methotrexate suppress inflammation? Clin Exp Rheumatol 2010; 28 (5 Suppl 61): S21-3.

46. Warren RB, Mrowietz U, von Kiedrowski R, et al. An intensified dosing schedule of subcutaneous methotrexate in patients with moderate to severe plaque-type psoriasis (METOP): a 52 week, multicentre, randomised, double-blind, placebocontrolled, phase 3 trial. Lancet 2017; 389: 528-37.

47. Peng Z, Fernandez P, Wilder T, et al. Ecto-5'-nucleotidase (CD73)-mediated extracellular adenosine production plays a critical role in hepatic fibrosis. Nucleosides Nucleotides Nucleic Acids 2008; 27: 821-4.

48. Peng Z, Borea PA, Varani K, et al. Adenosine signaling contributes to ethanol-induced fatty liver in mice. J Clin Invest 2009; 119: 582-94.

49. Aithal GP. Hepatotoxicity Related to Methotrexate. In: DrugInduced Liver Disease (Third Edition). Kaplowitz N, DeLeve LD (eds). Academic Press, Boston 2013; 593-604.

50. O'rourke RA, Eckert GE. Methotrexate-induced hepatic injury in an adult: a case report. Arch Intern Med 1964; 113: 191-4.

51. Shetty A, Cho W, Alazawi W, Syn WK. Methotrexate hepatotoxicity and the impact of nonalcoholic fatty liver disease. Am J Med Sci 2017; 354: 172-81.

52. Kalb RE, Strober B, Weinstein G, Lebwohl M. Methotrexate and psoriasis: 2009 National Psoriasis Foundation Consensus Conference. J Am Acad Dermatol 2009; 60: 824-37.

53. Nast A, Jacobs A, Rosumeck S, Werner R. Methods Report: European S3-Guidelines on the systemic treatment of psoriasis vulgaris - update 2015 - EDF in cooperation with EADV and IPC. J Eur Acad Dermatol Venereol 2015; 29: e1-22.

54. Tilling L, Townsend S, David J. Methotrexate and hepatic toxicity in rheumatoid arthritis and psoriatic arthritis. Clin Drug Investig 2006; 26: 55-62.

55. Lindsay K, Gough A. Psoriatic arthritis, methotrexate and the liver - are rheumatologists putting their patients at risk? Rheumatology 2008; 47: 939-41. 
56. Wenk K, Arrington K, Ehrlich A. Psoriasis and non-alcoholic fatty liver disease. J Eur Acad Dermatol Venereol 2011; 25: 383-91.

57. An J, Zhang D, Wu J, et al. The acitretin and methotrexate combination therapy for psoriasis vulgaris achieves higher effectiveness and less liver fibrosis. Pharmacol Res 2017; 121: 158-68.

58. Szepietowski J, Adamski Z, Chodorowska G, et al. Treatment of psoriasis vulgaris: guidelines of experts of the Polish Dermatological Society. Part II: moderate to severe psoriasis. Dermatol Rev 2014; 101: 455-72.

59. Schmitt J, Rosumeck S, Thomaschewski G, et al. Efficacy and safety of systemic treatments for moderate-to-severe psoriasis: meta-analysis of randomized controlled trials. Br J Dermatol 2014; 170: 274-303.

60. Garber C, Plotnikova N, Au S, et al. Biologic and conventional systemic therapies show similar safety and efficacy in elderly and adult patients with moderate to severe psoriasis. J Drugs Dermatol 2015; 14: 846-52.

61. Rossi RE, Parisi I, Despott EJ, et al. Anti-tumour necrosis factor agent and liver injury: literature review, recommendations for management. World J Gastroenterol 2014; 20: 17352-9.

62. Stanley TL, Zanni MV, Johnsen S, et al. TNF-alpha antagonism with etanercept decreases glucose and increases the proportion of high molecular weight adiponectin in obese subjects with features of the metabolic syndrome. J Clin Endocrinol Metab 2011; 96: E146-50.

63. Burska AN, Sakthiswary R, Sattar N. Effects of tumour necrosis factor antagonists on insulin sensitivity/resistance in rheumatoid arthritis: a systematic review and meta-analysis. PLoS One 2015; 10: e0128889.

64. Gisondi P, Galvan A, Idolazzi L, Girolomoni G. Management of moderate to severe psoriasis in patients with metabolic comorbidities. Front Med 2015; 2: 1.

65. Feagins LA, Flores A, Arriens C, et al. Nonalcoholic fatty liver disease: a potential consequence of tumor necrosis factorinhibitor therapy. Eur J Gastroenterol Hepatol 2015; 27: 1154 60.

66. Di Minno MND, Iervolino S, Peluso R, Russolillo A, Lupoli R, Scarpa R, et al. Hepatic steatosis and disease activity in subjects with psoriatic arthritis receiving tumor necrosis factorblockers. J Rheumatol 2012; 39: 1042-6.

67. Llamas-Velasco M, Concha-Garzón MJ, García-Diez A, Daudén E. Liver injury in psoriasis patients receiving ustekinum$a b$ : a retrospective study of 44 patients treated in the clinical practice setting. Actas Dermosifiliogr 2015; 106: 470-6. 